
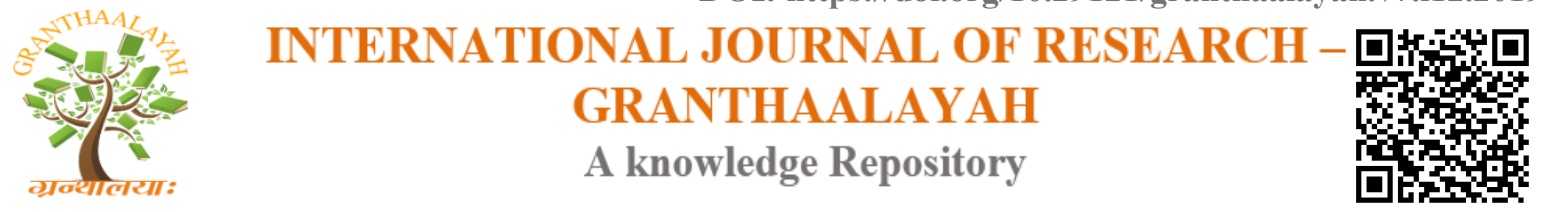

Science

\title{
HIGH-SPEED QUECHING OF HIGH CARBONSTEEL
}

\author{
Bello Imamudeen ${ }^{1}$, Shiv Kumar Singh ${ }^{2}$ \\ ${ }^{1}$ Department- Welding and Fabrication, Kano State Polytechnic \\ ${ }^{2}$ Department of Physics, Federal University of Kashere, Gombe State, Nigeria
}

\begin{abstract}
Medium and high carbon steels are usually quenched in polymer and oil in order to avoid cracking and distortion; however, recent studies have proved that it is possible to minimize cracking using water as a coolant of these steels by promoting extremely high cooling rates. By great agitation and velocity of quench ant, the vapour blanket is reduced or prevented during water quench, allowing uniform hardening of the surface. In this study, the cooling severity of a spray quenching system and a high-speed quenching chamber are studied. Cylindrical samples of AISI 304 stainless steel (20mm in diameter and $100 \mathrm{~mm}$ length) were employed to characterize the cooling severity. Thermal data was acquired through K-type thermocouples placed in the sample at three positions, $1 \mathrm{~mm}$ below surface, mid-radius and at centre of the specimen, connected to a data acquisition system. High thermal gradients were observed in both systems, being the high-speed chamber the severest cooling. The maximum cooling rate obtained at the surface was 470 and $300 \mathrm{C} / \mathrm{s}$ for the high-speed chamber and the spray system, respectively. In addition, 5160 spring steel samples were quenched for short times in both systems; the cooling was interrupted to avoid through transformation and to produce a case-core type microstructure. Different cooling times were used for the interrupted quenching to modify the marten site case thickness. No cracks.
\end{abstract}

Keywords: Quenching; Acquisition; High-Speed Quenching.

Cite This Article: Bello Imamudeen, and Shiv Kumar Singh. (2019). "HIGH-SPEED QUECHING OF HIGH CARBONSTEEL." International Journal of Research - Granthaalayah, 7(12), 25-31. https://doi.org/10.29121/granthaalayah.v7.i12.2019.297.

\section{Introduction}

The automotive industry is continuously seeking to enhance steel properties; at the same time, it aims to reduce costs and make the processes eco-friendly. Medium carbon alloys are susceptible to distortion and cracking; therefore, medium and slow cooling rates are desired and obtained by the use of oil-quenching or polymer quenching. The most common techniques are immersion quenching, usually using agitation, and spray quenching with a single fluid or a mixture [1-3]. During quenching, thermal and transformational stresses arise and might cause distortion or cracking if they exceed the material's strength [4]. By inducing very high cooling rates during the quenching, it is possible to reduce the distortion and cracking if the quench is interrupted prior to 
marten site transformation at the core. High cooling rates are usually obtained with water or brine quench ants [5]. In this paper, a comparison between a spray array and high-speed quenching using water is analysed to determine the cooling condition and the time that produces a marten site case on AISI 5160 spring steel.

\section{Experimental Procedure}

A probe of an AISI-304 austenitic stainless steel [6] was used to obtain thermal data and to characterize the system and determine cooling severity. The probe's length to-thickness ratio was 5:1 $(100: 20 \mathrm{~mm})$. Three $\varnothing 1 \mathrm{~mm}$ blind holes were drilled by electro discharge machining up to the mid-length of the probe. They were drilled in the mid-thickness plane, one in the geometric centre (T3), $1=4$ thickness (T2), and $1 \mathrm{~mm}$ below the surface (T1). Three ungrounded $\varnothing 1 \mathrm{~mm}$ Type-K thermocouples were inserted into the holes until the half of the body. The holes were machined in order to tightly adjust the thermocouples. A small amount of graphite powder was deposited into the holes before thermocouple insertion to ensure good contact between the thermocouple and the probe. After thermocouple placement, a ceramic coating was used to seal the holes to prevent the quench ant from penetrating into the holes. The thermocouples were differentially connected (negatives were connected to different channels not to ground) using a 75KX resistor between the negative terminal and ground to provide a good reference. Data was acquired at a frequency of $100 \mathrm{~Hz}$. The specimens were heated at 900C and held in the furnace until a homogenous temperature in the sample was attained. All experiments were performed with a quench ant temperature of $25 \mathrm{C}$, and there was no temperature increase of the quench ant because of the large reservoir (600 LTS). After a uniform temperature was achieved, the sample was placed on the spray quenching (SQ) system (transfer time<3s). The spay system includes four full ova cone nozzles. The nozzle features are shown in Table 1. a schema of the SQ system showing the working distance, probe size, and holder. Furthermore, a high-speed quenching (HSQ) system was built. The hot sample is placed on the holder which is has a pneumatic cylinder. The cylinder is PLC activated and lifts the sample inside the chamber. The chamber is filled with a high speed water flow in less than $2 \mathrm{~s}$. The sample remains fully immersed while water passes through the chamber . Parameters of HSQ are shown on Table 2. Cooling curve, cooling curves rates and recalescence were determined for both systems at different times. AISI 5160 cylindrical samples $20 \mathrm{~mm}$ in diameter and 100mm length were quenched in the HSQ system. The chemical composition of the steel is displayed in Table 3. The samples were austenitized at 850C. Different interruptedquenching times were employed to obtain different combinations of microstructures in the cylinders

Table 1: Spray parameters used for tests.

\begin{tabular}{|l|c|c|c|c|}
\hline TYPES & Spray angle & Water (1/min) & Pressure (bars) & Temp Quench art \\
\hline FULL OVAL CONE & $90^{\circ}$ & +9.3 & 7 & $25^{\circ} \mathrm{C}$ \\
\hline
\end{tabular}

Spray Water Pressure Temperature Type Angle (1/min) (bars) Quench ant Full oval cone 9069.37 $25 \mathrm{C}$

(Marten site at the surface and binate at the core). After the HSQ was conducted, some samples were tempered in order to allow the bainitic transformation. The different quenching and tempering conditions are shown on Table 4 . The tempering time was $1 \mathrm{~h}$. After the quenching and 
tempering, samples were prepared for metallographic inspection. Furthermore, the samples were characterized by means of scanning electron microscope (SEM) and X-ray diffraction (XRD).

\section{Results and Discussion}

\subsection{Quenching Severity}

The cooling curves of the SQ system. The temperature of the sample became stable at room temperature after $20 \mathrm{~s}$ of quenching. The HSQ system displayed a more aggressive cooling than the SQ system. The cooling rate curves corresponding to the SQ and HSQ system. The highest cooling rate obtained in the SQ system was 300C/s at the surface of the sample, while for HSQ, it was 470C. The surface temperature was calculated using a parabolic temperature distribution [7]. In the latest, the sample became stable at room temperature around $15 \mathrm{~s}$ of water quenching. High thermal gradients were achieved in the HSQ system, and this allowed the formation of a case-core type microstructure.

\subsection{Quenching of Spring Steel}

The AISI 5160 samples were quenched in the HSQ system according to Table 4 conditions. The thermal process is illustrated in Fig. 3. The quenching was interrupted in all tests to prevent through hardening. As a consequence of the thermal gradients achieved and the temperature distribution presented for this system, a case-core type microstructure was presented for most conditions. The cooling curves and the temperature distribution for different tests are shown in Fig. 4. Samples showed a thermal recovery after the interruption of the quenching. This produced a self-tempering of the martensitic case at the surface, where the temperature drops below Ms. The SEM micrographs obtained from the samples 2Q and 4Q are shown in Fig. 5. Even though the quenching was interrupted in all tests, the samples $2 \mathrm{Q}, 4 \mathrm{Q}$.

Table 2: Parameters used at the HSQ tests.

\begin{tabular}{|c|c|c|}
\hline Flow rate (1pm) & Pressure (bar) & Temp Quench ant \\
\hline 1325 & 4 & $25^{\circ} \mathrm{C}$ \\
\hline
\end{tabular}

Table 3: Chemical composition of the steel.

\begin{tabular}{|c|c|c|c|c|c|c|c|c|c|}
\hline $\mathbf{C}$ & $\mathbf{M n}$ & $\mathbf{S}$ & $\mathbf{S i}$ & $\mathbf{N i}$ & $\mathbf{C r}$ & $\mathbf{C u}$ & $\mathbf{N b}$ & $\mathbf{T i}$ & $\mathbf{A l}$ \\
\hline 0.58 & 0.017 & 0.016 & 0.26 & 0.01 & 0.78 & 0.013 & 0.003 & 0.0038 & 0.0264 \\
\hline
\end{tabular}

Table 4: Q \& T process applied to the spring steel.

\begin{tabular}{|c|c|c|}
\hline Sample ID & HSQ & Tempering \\
\hline $2 \mathrm{Q}$ & $2 \mathrm{~S}$ & - \\
\hline $4 \mathrm{Q}$ & $4 \mathrm{~S}$ & - \\
\hline $6 \mathrm{Q}$ & $6 \mathrm{~S}$ & - \\
\hline $8 \mathrm{Q}$ & $8 \mathrm{~S}$ & - \\
\hline $1 \mathrm{~T}$ & $1 \mathrm{~S}$ & $250^{\circ} \mathrm{C}$ \\
\hline $2 \mathrm{QT}$ & $2 \mathrm{~S}$ & $250^{\circ} \mathrm{C}$ \\
\hline $3 \mathrm{QT}$ & $3 \mathrm{~S}$ & $250^{\circ} \mathrm{C}$ \\
\hline
\end{tabular}


Sample ID HSQ Tempering

2Q 2s-4Q 4s-6Q 6s-8Q 8s - 1QT 1s 250C 2QT 2s 250C 3QT 3s 250C 4QT 4s 250C $5 \mathrm{QT} 5 \mathrm{~s} 250$

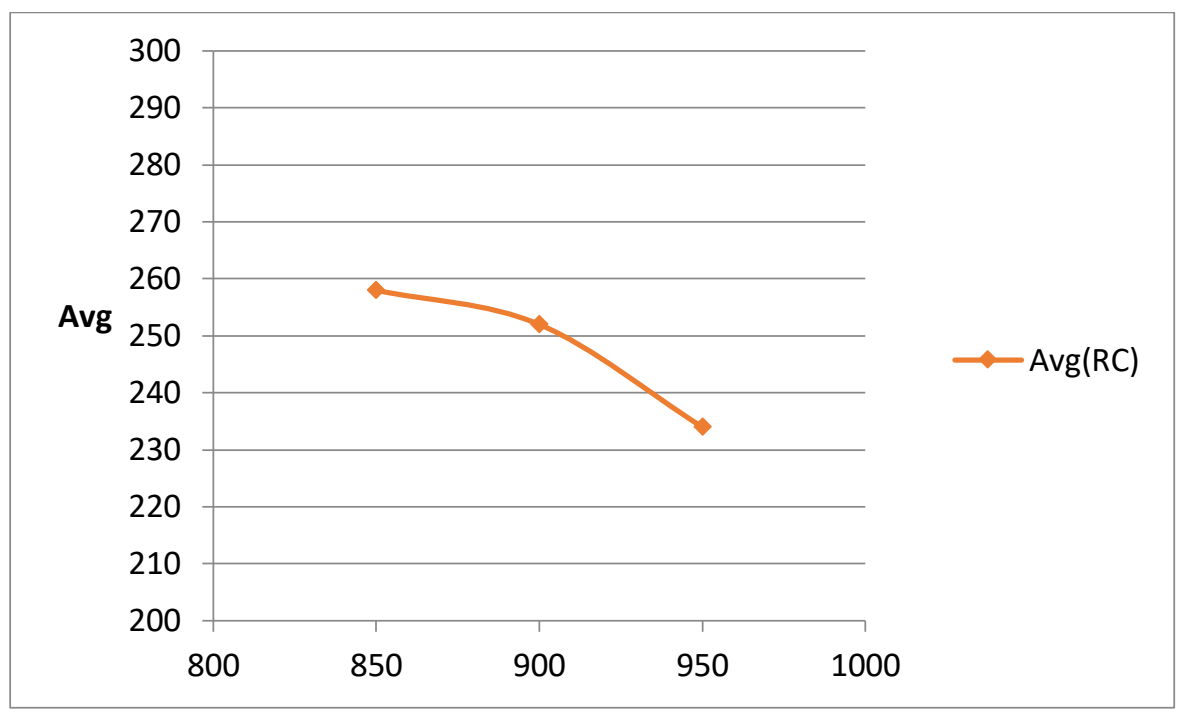

Figure 1: cooling curves showing the temperature distribution of the SQ system.

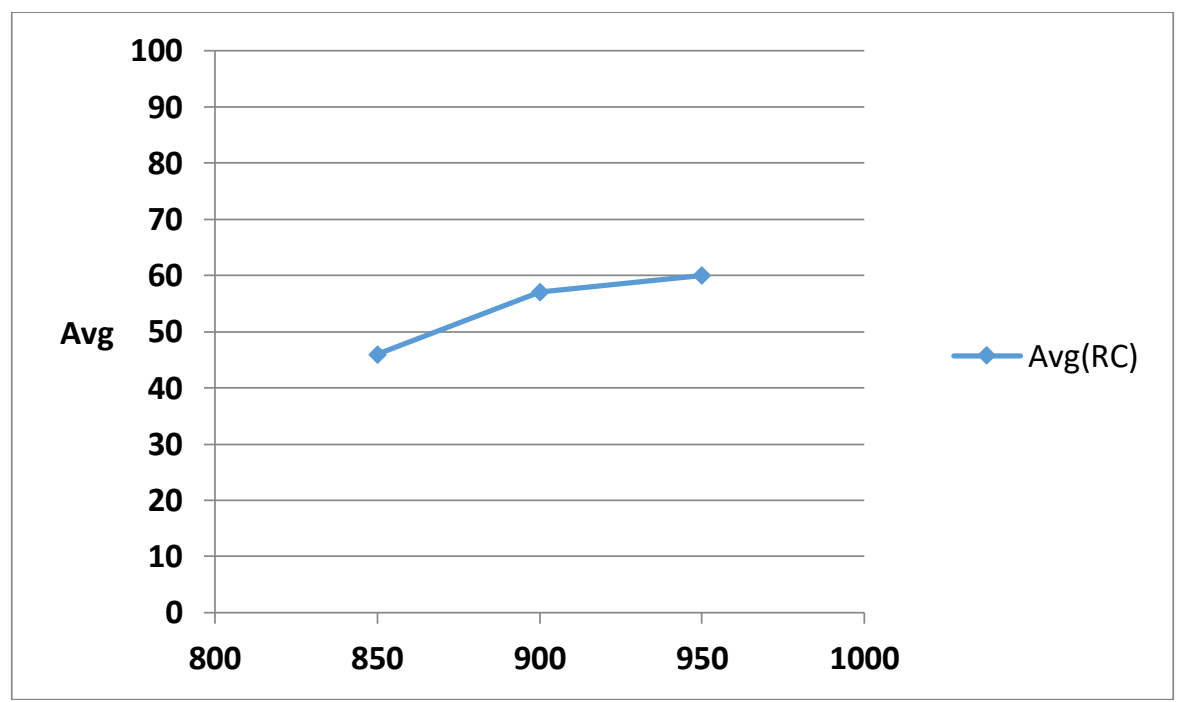

Figure 2: Cooling curves showing the temperature distribution of the HSQ system.

6Q, and 8Q (air cooling after the interrupted quenching) were through hardened due the high hardenability of this steel. The sample 4Q presented the highest amount of retained austenite at the core of the sample. The core temperature was hold above the Ms (247C). Therefore, the samples after the time-quench were placed in a furnace at $250 \mathrm{C}$ for $1 \mathrm{~h}$ to let the bainitic transformation to take place, avoiding through hardening, and to reduce the amount of retained austenite at the core. For the sample 1QT, the temperature became stable at 486C, the surface temperature did not cross the Ms, and the core went directly to the ferrite-pearlite nose. Therefore, a mixture of ferrite and pearlite colognes was obtained on the entire sample; this microstructure can be appreciated on 
Figs. 10(a) and 10(b). The same behaviour of the sample 1QT was presented in the sample 2QT in which temperature became stable at 330C and went into the Upper Bainite (UB).

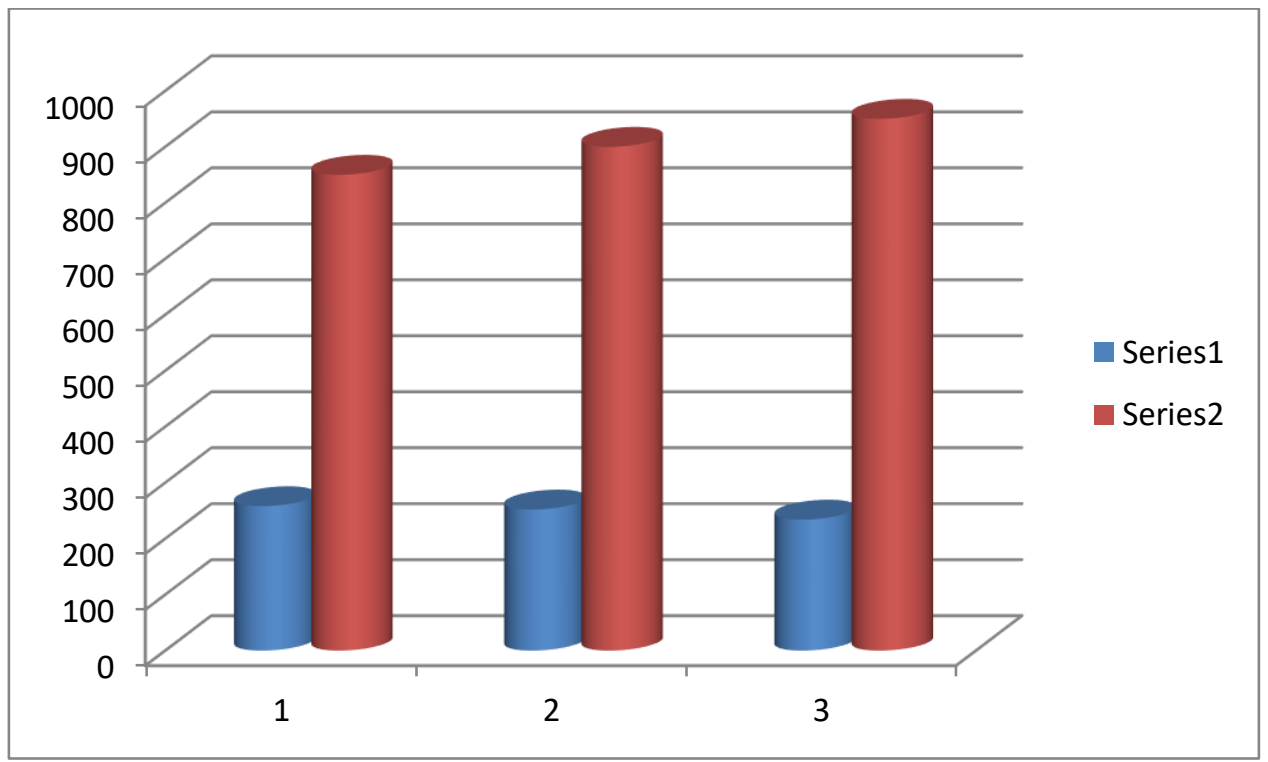

Figure 3: Thermal process applied to five samples after quenched.

Zone. In the samples 3QT and 4QT, tempered marten site (TM) was observed at the surface of the samples since superficial zones reached the Ms during the cooling process. The temperature of these samples became stable at 300 and 247C, respectively; therefore, Lower Bainite (LB) was obtained at the core of those samples, and with the sample 4QT being the one which presented the best combination with a higher thickness of the marten site case. This is shown in Figs. 10(c) and $10(\mathrm{~d})$. The temperature of sample 5QT became stable at 215C; as a consequence, a mixture of TM and LB at the core of the sample was observed on the SEM analysis. The corresponding XRD patterns of the samples quenched at the HSQ system for 2, 4, 6, and 8s followed by air cooled. Even there were high.

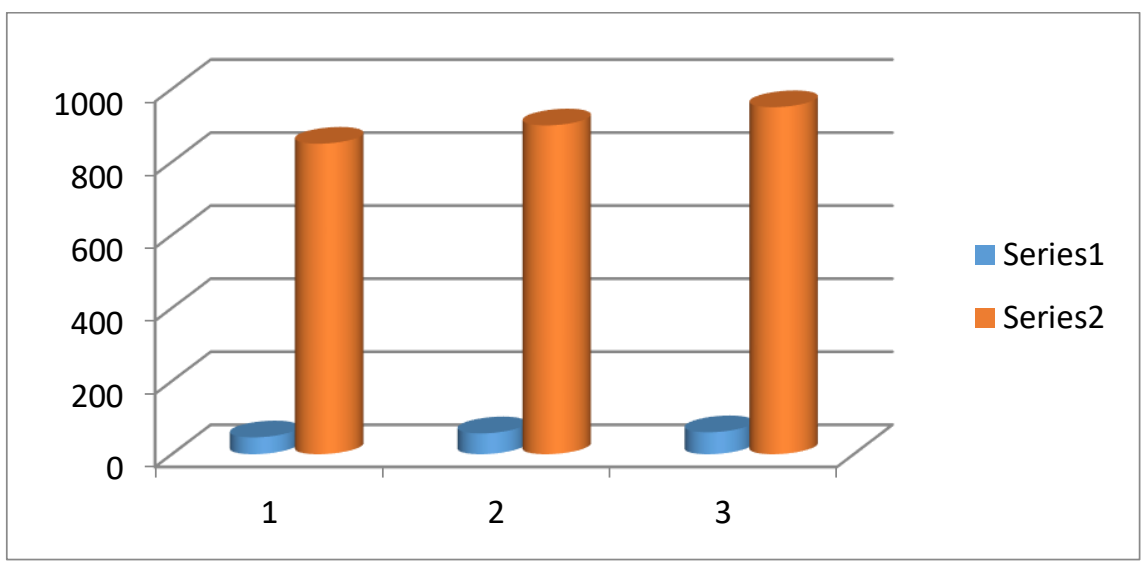

Figure 4: Cooling curves obtained from the samples quenched for (a) $1 \mathrm{~s}$, (b) $2 \mathrm{~s}$, (c) $3 \mathrm{~s}$, (d) 4s, and (e) $5 \mathrm{~s}$. 
Thermal gradients within the samples, due to the high hardenability of this steel, most of the microstructure observed in the XRD patterns were tempered marten site (TM). Little retained austenite (RA) was noticed in the XRD patterns. Samples heat treated as described before manifested TM at the surface and a mixture of TM and presence of RA at the core, with the sample 4Q being the one that presented the highest intensity of the RA fcc (200c) peak. The XRD patterns of the quench and tempered samples. Samples 1QT and 2QT showed a well-defined (110a) peak with higher intensity than the other three samples. This corroborates the presence of ferrite-pearlite at the core of this samples, which were indexed accordingly to this phases [8].

A particular behaviour was observed on the strongest line of the (110a) peak, increasing the quenching time from 1 to $5 \mathrm{~s}$; the intensity of this peak decreased, the width of the peak had a slight increment, and the peak shifted to the left with respect to the original peak related with the Fea. This behaviour can be explained with the presence of a lattice deformation, presence of residual stresses, or defect concentration [9]. The XRD patterns for the samples 3QT and 4QT indicated the presence of bainites at the core and the pattern corresponding to the sample 5QT showed the highest distortion of the Fea principal peak and displayed match with the Marten site indexed card.

\section{Conclusions}

Spray quenching and high speed systems were compared to determine the cooling severity of the systems. The high speed system analysed was the severest cooling system and the best option to obtain high thermal gradients, high cooling rates, and to produce case-core microstructure in small samples. Samples of AISI 5160 spring steel were quenched and tempered and the results were characterized by means of SEM and XRD. It was possible to obtain high thermal gradients that produce a case-core type microstructure using water as quench ant and at the same time avoid quench crack on a $0.6 \mathrm{wt}$. \% $\mathrm{C}$ steel. The presence of retained austenite at the core of the samples was avoided a by applying an interrupted quenching followed by a proper tempering, promoting the bainitic transformation. The highest value of retained austenite was observed on the sample quenched by $4 \mathrm{~s}$ at the high speed quenching system and air cooled. Tempered marten site at surface and bainitic core were obtained in the samples quenched 3 and $4 \mathrm{~s}$, before tempering, which according to the XRD patterns were the samples which had the most deformation, a sign of the presence of compressive residual stresses.

\section{References}

[1] Totten, G. E., Bates, C. E., and Clinton, N. A., Other Quenching Processes, Handbook of Quenchants and Quenching Technology, ASM International, Materials Park, OH, 1993.

[2] Liscic, B. and Filetin, T., "Measurement of Quenching Intensity, Calculation of Heat Transfer Coefficient and Global Database of Liquid Quenchants," Mater. Eng., Vol. 19, 2012, pp. 52-63.

[3] Scott, H., The Problem of Quenching Media for the Hardening of Steel, Heat Transfer Symposium, ASM International, Materials Park, OH, 1933.

[4] Liscic, B., Tensi, H. M., Canale, L.C. F., and Totten, G. E., Quenching Theory and Technology, 2nd ed., CRC Press, Boca Raton, FL, 2010.

[5] Kobasko, N. I., Arononov, M. A., Powell, J. A., and Totten, G. E., "Intensive Quenching Systems: Engeneering and Desing," ASTM International, West Conshohocken, PA, 2010.

[6] ASM International, Properties and Selection: Irons Steels and High Performance Alloys, ASM Handbook, Vol. 1, ASM International, Materials Park, OH, 1990. 
[7] Lozano, D. E., Mercado-Sol'1s, R. D., Cola's, R. Canale, L. F., and Totten, G. E., "Surface Temperature and Heat Transfer Coefficient Determination During Quenching for Martensite Fraction Prediction Using a Parabolic Heat Transfer Model," Proceedings of the 6th International Quenching and Distortion Control Conference, Chicago, IL, Sept 9-13, 2012, pp 746-754.

[8] Swanson, H. E., Fuyat, R. K., and Ugrinic, G. M., "X-Ray Diffraction Powder Patterns," Circ. 539, The National Bureau of Standards, Gaithersburg, MD, 1955.

[9] Cullity, B. D., Elements of X-Ray Diffraction, 3rd ed., Addison-Wesley, Boston, MA, 1956.

*Corresponding author.

E-mail address: snghshvkmr@ yahoo.co.in 